\title{
AN EFFICIENT NEW METHOD ON ACCURATELY ESTIMATING GALILEO VPL
}

\author{
Ying Guo ${ }^{1,2}$, Xiushan $\mathrm{Lu}^{1}$ \\ ${ }^{I}$ Geoinformation Science \& Engineering College, Shandong University of Science and \\ Technology, Qingdao, China 266510 \\ 2 The Geomatics and Applications Laboratory, Liaoning Technical University, Liaoning, \\ FuXin, China 123000
}

Abstracts: The future GALILEO system will use multi-frequency data to process, which may eliminate preferably the influence of ionosphere relay. So the VPL estimation is mainly to consider how to efficiently use the satellite Signal-InSpace (SISE). To efficiently utilize SISE in GNSS and to rationally estimate the user Vertical Protection Level, it is the key to ensure normal application of GNSS and user's safety. For the investigation requirement of GALILEO VPL algorithm. Based on the character which SISE influences on users' positioning, this paper provides an estimating model of equivalent range error of SISE which influences users efficiently. On the bases of the integrity theory of VPL and the principle of synthetizing and assessing the uncertainty of measurement errors, it also gives a new algorithm to estimate VPL rationally to provide references for the development and the application of the integrity theories of GNSS.

Keywords: Galileo; integrity theory; SISE; VPL

\section{INTRODUCTION}

The satellite broadcast ephemeris error is an important influence on the reliability of user's positioning and the safety of user's life. It includes the orbit error and the clock error, which is named as Signal-In-Space (SISE). GNSS enhances the reliability of the satellite broadcast ephemeris by predicting and monitoring SISE integrity (Veit Oehler, 2004; Carlos Hernandez Medel, 2002, 2005).

Guo, Y. and Lu, X., 2008, in IFIP International Federation for Information Processing, Volume 258; Computer and Computing Technologies in Agriculture, Vol. 1; Daoliang Li; (Boston: Springer), pp. 231-238. 
User applies the integrity parameters of SISE, which are broadcasted by GNSS, and local integrity information, which affects on the broadcast of satellite signal, for example, delay correction and correct error of ionosphere and troposphere, receiver noise, multi-path effect and so on, to estimate user vertical protection level (VPL) to predict and monitor the safety state of navigation system (J.T. Wu, 2002; Curtis A. Shively, 2005; Stephane, 2002). If it is estimated more than nominal range, VPL includes some false data, and reduces the integrity function of system; If it is less, it leads to some mislead data to influence normal application of system. So the ideal VPL algorithm is the key to accurate application of the navigation system.

The appearance of the integrity theory of the GALILEO system, which has its own characteristics, improves the integrity theory of GNSS. Because the integrity principle of the GALILEO SISE varies from that of EGNOS/WAAS UDRE (User Different Range Error), which is used to name as satellite ephemeris error, the VPL algorithm of the two systems are also different. At present, the estimating method of GALILEO VPL investigated by ESA is kept secret. Based on the algorithm of EGNOS/WAAS VPL, Stephane and Bruno in reference (Stephane, 2002) provide two selective methods under the assumed condition that the satellite exists in the two cases.

Aim at the need of the development and the application of the integrity theories of GALILEO system and GNSS, this paper studies the estimating formula of equivalent range error of SISE which influences users efficiently. On the bases of the integrity theory of VPL, and the principle of synthetizing and assessing the uncertainty of measurement errors, it further provides a new method of estimating VPL and illustrates its reliability.

\section{PRINCIPLE OF ESTIMATING VPL}

Based on the linear model of user position estimation

$y=G x+\varepsilon$

Here

The vector $y$ is the vector of observed differences between the receiver measured pseudo range, and the distance between satellite and initial known approximate user position. $G$ is the geometry metric consisting of one row for each monitored satellite in the view. The 4-vector $x$ represents user position (east, north, up) and clock bias, $\varepsilon$ represents the variance metric of error term.

According to the least-square law, weight of remain-covariance and assessed principle of measurement uncertainty to compute VPL. Its primary model is 


$$
\begin{aligned}
& V P L=K_{v p l} \sqrt{\sum_{i=1}^{n} S_{u p, i}^{2} \cdot \sigma_{i}^{2}} \\
& \sigma^{2}{ }_{i}=\sigma_{i, S I S E}^{2}+\sigma_{i, \text { ion }}^{2}+\sigma_{i, \text { trop }}^{2}+\sigma_{i, m p+n o i s e}^{2} \\
& S=\left(G^{T} W^{-1} G\right)^{-1} G^{T} W \\
& S=\left(S_{\text {east }}, S_{\text {north }}, S_{\text {up }}, S_{\text {clock }}\right)
\end{aligned}
$$

Where

$K_{v p l}$ is the coefficient factor which is estimated by VPL, $W$ is the errorcovariance weighting metric, $\sigma_{i}$ represents the total mean error which No. i satellite arouses user position, including $\sigma_{i, S I S E}$, which is the mean error of SISE, $\sigma_{i, i o n}$ which is the remain mean error of ionosphere delay correction, $\sigma_{i, t r o p}$ which is the remain mean error of troposphere delay correction, and $\sigma_{i, m p+n o i s e}$ which the mean error of receiver noise and multi-path effect.

Because the error between SISE and other local factors are relative independent, their effects are considered as the simple accumulation of each part. And SISE of formula is computed in term of mean-error.

\section{EXISTING VPL ALGORITHM}

Formula (2) is the primary model of VPL, and is the usual one that is used to estimate VPL of EGONS and WAAS. Based on that model, both Stephane and others provide two selective algorithms:

Algorithm 1: the VPL estimating algorithm based on WAAS/EGNOS (Stephane, 2002)

$$
V P L=\sum_{i_{S A T}}\left|M\left(3, i_{\text {sat }}\right) * S I S E\right|+K_{V P L} \sqrt{\left(G^{T} W_{u-l o c a l}^{-1} G\right)^{-1}(3,3)}
$$

Here

$$
\begin{aligned}
& M=\left(G^{T} W^{-1} G\right)^{-1} G^{T} W^{-1} \\
& W_{u-\text { local }}=\left[\begin{array}{lrrr}
\sigma_{u-l o c a l, R X}(1)^{2} & 0 & \ldots & 0 \\
0 & \cdots & \ldots & 0 \\
0 & 0 & \sigma_{u-l o c a l, R X}(i)^{2} & 0 \\
0 & 0 & \cdots & \sigma_{u-\text { local }, R X}(n)^{2}
\end{array}\right] \\
& \sigma_{u-\text { local }, R X}^{2}\left(i_{\text {sat }}\right)=\sigma_{i, \text { ion }}^{2}+\sigma_{i, \text { trop }}^{2}+\sigma_{i, \text { mp }+ \text { noise }}^{2}
\end{aligned}
$$


Where

$W_{u-\text { local }}$ represents the error-covariance metric of user location, $\sigma_{u-l o c a l}\left(i_{\text {sat }}\right)$ represents the total error of No. i satellite, except SISE.

The assumed condition of formula (4) is that all satellites in the view are in the state of WUL, the probability seldom occurs. And the errors which both SISE and others effect on are estimated respectively first, and then added together. So, Stephane studies another algorithm again.

Algorithm 1: the estimating model of GALILEO VPL is expressed as

$$
\begin{aligned}
& V P L=\max _{i_{s a t}}\left\{\left|M_{u}\left(3, i_{s a t}\right) * S I S E\right|+K_{V P L} \sqrt{\left(G_{u}^{T} W_{u}^{-1} G_{u}\right)^{-1}(3,3)}\right\} \\
& W_{u}=\left[\begin{array}{lrlll}
\sigma_{u, R X}(1)^{2} & 0 & \ldots & 0 & \\
0 & \ldots & \ldots & 0 \\
0 & 0 & \sigma_{u-\text { local }, R X}(i)^{2} & 0 \\
0 & 0 & \cdots & \sigma_{u, R X}(n)^{2}
\end{array}\right] \\
& \sigma_{u, R X}^{2}(i)=\sigma_{i}^{2}
\end{aligned}
$$

This method is assumed that only one satellite is located in WUL. SISE of this satellite is solely computed the influence, others are created weighting metric together with local factors. In the formula of the weighting, SISE is computed in term of mean-error. But broadcast SISE is estimated with uncertainty principle, and user can not obtain its error. So, this method need to be fully studied and illustrated its reliability.

\section{A NEW ESTIMATING VPL METHOD}

In the user navigation system, there is only certain probability of the both assumed conditions of the above two algorithms and it can not generalize all cases better. Algorithm 1 respectively considers SISE and other effects, and the final effect is considered to be adding up simply to increase the error of positioning; Algorithm 2 needs to provide mean-error of SISE.

Based on the satellite positioning law, the uncertainty law of measurement error, and the integrity theory of VPL, the equivalent range error of SISE accurately effecting user is analyzed, and a method of rational computing VPL is provided, which is as follows.

\subsection{Accurate Equivalent Range Error of SISE}

In GALILEO system, SISE, which includes predicting parameter SISA and monitoring parameter SISMA, is the value that the satellite locates in 
WUL. So, at one epoch, in the location of user receiver, all satellites in the view can not be located in WUL at the same time, and can not ensure that only one or some locate in WUL. But, at this time, both satellite position and SISE may be obtained, and the position of the user receiver may be approximately computed. So, the angle between the SISE vector direction and the one form satellite to receiver may be computed, furthermore, $S I S E_{A C}$ which is the equivalent range error of SISE effecting on user may computed, its estimating program is following.

Because satellite clock error has the same influence on all users in view, but the influence that the satellite orbit error affects varies with different location. These two errors will be respectively considered.

$\left(S_{x}, S_{y}, S_{z}\right)$ are assumed as predicted vector parameter of satellite orbit error, $b_{S}$ is its scale, and its unit vector is $e_{S V}=\left(e_{x}, e_{y}, e_{z}\right), b_{\text {clock }}$ represented satellite clock error, the vector form satellite to user is $e_{S V-u}=\left(e_{x, u}, e_{y, u}, e_{z, u}\right)$. So the angle $\vartheta$ between $e_{S V}$ and $e_{S V-u}$ is computed by

$\vartheta=\operatorname{arc}\left(e_{S V} \bullet e_{S V-u}\right)$

$b_{A C}$ is accurate equivalent range error created by satellite orbit error, is expressed by

$b_{A C}=b_{S} \cdot \cos \vartheta$

Assumed that the observable vector $G^{i}$ is expressed by

$G^{i}=\left(G_{1}^{i}, G_{2}^{i}, G_{3}^{i}, 1\right)$

So $b_{A C}$ is

$$
b_{A C}=\sqrt{\left(G_{1}^{i} \cdot S_{x}\right)^{2}+\left(G_{2}^{i} \cdot S_{Y}\right)^{2}+\left(G_{3}^{i} \cdot S_{Z}\right)^{2}}
$$

That is to say, SISE $E_{A C}$ is represented as

$$
S I S E_{A C}=\sqrt{b_{A C}^{2}+b_{\text {clock }}^{2}}
$$

VPL is computed with $S I S E_{A C}$ without considering the position of satellite at some epoch. This estimating method possesses preferable applicability and reliability.

\subsection{Estimating VPL}

Based on the relative principles, this paper introduces into $\operatorname{SISE}_{A C}$, estimating VPL model of GALILEO system is expressed by 


$$
V P L=\sqrt{\sum_{i=1}^{n}\left(S_{u p, i}^{2} \cdot \sigma_{G, i}^{2}\right)}
$$

Here

$$
\sigma_{G, i}^{2}=\operatorname{SISE}_{A C}^{2}+K_{v p l}^{2} \cdot\left(\sigma_{\text {iono }, i}^{2}+\sigma_{\text {trop }, i}^{2}+\sigma_{m p t h, i}^{2}\right)
$$

\section{CASE STUDY}

It is rational in theory to estimate GALILEO VPL based on equivalent range error accurately effect on SISE, furthermore to illustrate feasibility and reliability by simulating data.

\subsection{Data Source}

(1) Ephemeris datum: They are broadcast ephemeris of Sept. 19-21 in 2005, which are updated with the software of BERNESE GPS, and the epoch interval is five minutes.

(2) SISE: Satellite orbit errors are obtained by updating and comparing broadcast ephemeris and precise one, and then transformed into the value of WUL. Clock error is described as $0.3 \mathrm{~m}^{[5]}$; the interval of epoch is $\mathrm{r} 5$ minutes, both SISE and $\sigma_{\text {SISE }}$ are estimated with existing methods.

(3) Positioning error: The relative parameters are obtained with formulas which are provided to compute remain of ionosphere and troposphere in the document [5]. The vertical grid ionosphere error is $0.7 \mathrm{~m}$.

\subsection{Simulated Result and Analysis}

According to the above-simulated data, the results which are estimated by algorithm 1 and the new illustrated method are described in the following figures. Where, series 1 represents the computed value with algorithm 1, and series 3 is estimated protect error.

By analyzing and comparing with different values (fig. 1), it can conclude that in the normal condition, VPL estimated by the new method is obviously less than that computed by algorithm 1 , and greatly more than the real error 


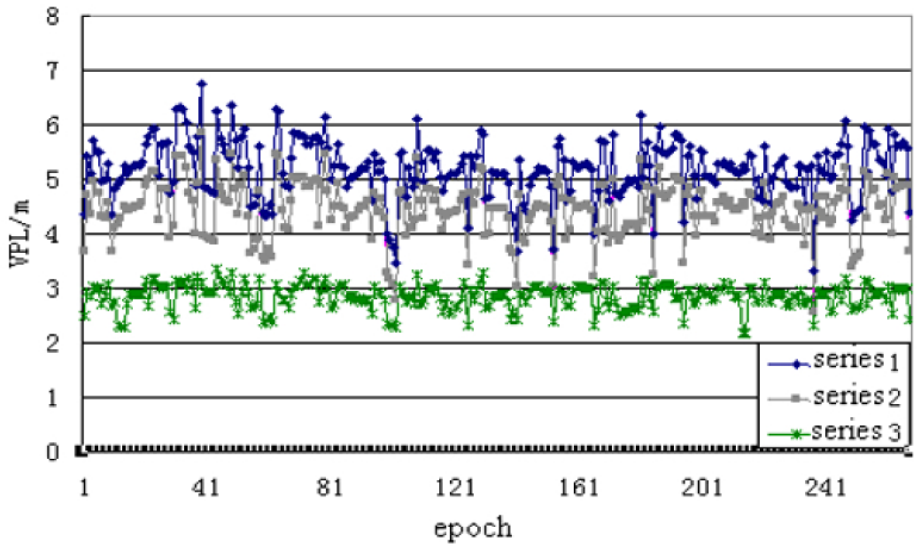

( a )

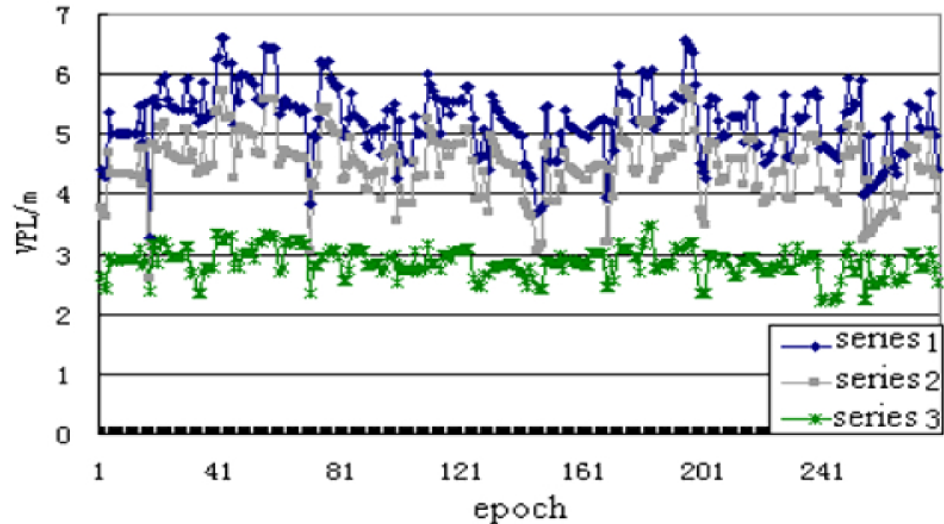

( b )

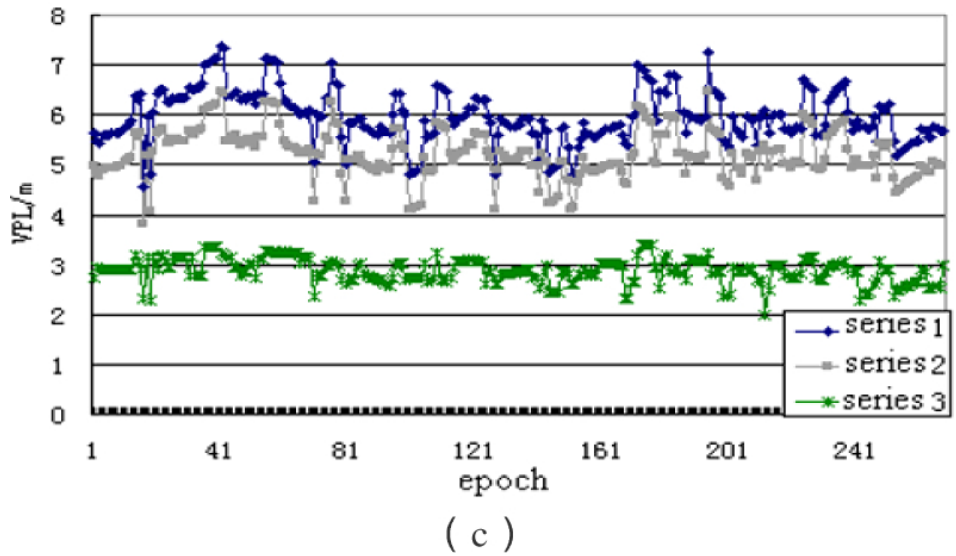

Fig. 1. The comparison of the VPL and VPE estimated by different algorithm 
of user positioning which is series 3. It indicates that this new algorithm is better than the existing one.

\section{CONCLUSION}

According to the investigating and practical need of VPL algorithm in GNSS and GALILEO system, on the basis of analyzing the existing GALILEO VPL, this paper provides an estimating model of equivalent range error of SISE in the positioning area according to the different influences that SISE has on with the differences of the users' locations. By the uncertainty principle of measurement errors and the integrity theory of VPL, it also gives a new rational GALILEO VPL algorithm, which is illustrated to be reliable with simulated data. So the conclusion is that this new algorithm is better than the existing ones.

\section{ACKNOWLEDGEMENT}

The worked reported in this paper is funded by Key Laboratory of Geo-informatics of State Bureau of Surveying and Mapping (200607) and Supported by Open Research Fund Program of the Geomatics and Applications Laboratory Liaoning Technical University (200628).

\section{REFERENCES}

Carlos Hernandez Medel. SISA Computation Algorithm and their applicability for Galileo Intergrity [J]. ION GNSS International Meeting of the Satellite Division, 24-27 Sept. 2002, Portland, OR. 2002, pp. 2173-2184.

Curtis A. Shively, Thomas T. Hsiao. Performance and Availability Anaysis of a Aimple Local Airport Position Domain Monitor for WAAS. ION GNSS International Meeting of the Satellite Division, 13-16 Sept. 2005, Long Beach, CA. 2005, pp. 2837-2854

Helmut Blomenhofer, Walter Ehret. Sensitivity Analysis of the Galileo Integrity Performance Dependent on the Ground Sensor Station Network [J]. ION GNSS International Meeting of the Satellite Division, 13-16 Sept. 2005, Long Beach, CA. 2005, pp. 2837-2854

J. T. Wu \& Stephen Peak. An Analysis of Satellite Integrity Monitoring Improvement for WAAS [J]. ION GNSS International Meeting of the Satellite Division, 24-27 Sept. 2002, Portland, OR. 2002, pp. 756-765.

Stephane Lannelongue, Bruno Lobert. On the Galileo User Integrity Computation [J]. ION GNSS International Meeting of the Satellite Division, 24-27 Sept. 2002, Portland, OR. 2002, pp. 1547-1556.

Veit Oehler. The Galileo Integrity Concept [J]. ION GNSS 17th International Meeting of the Satellite Division, 21-24 Sept. 2004, Long Beach, CA. 2004, pp. 604-615. 